Classification

Physics Abstracts

$77.90-81.20 \mathrm{~T}-81.40 \mathrm{R}-41.10 \mathrm{D}$

\title{
Pyroelectric transient torque. Theoretical and experimental study
}

\author{
J. C. Peuzin et M. Bottala \\ Division d'Electronique et de Technologie de l'Instrumentation, CEA-CENG-LETI-D. Opt.-CPM, \\ B.P. 85 X, 38041 Grenoble, France
}

(Reçu le 24 octobre 1989, révisé le 7 décembre 1989, accepté le 25 janvier 1990)

\begin{abstract}
Résumé. - Un échantillon pyroélectrique brusquement immergé dans un liquide diélectrique chauffé acquiert un moment dipolaire qui a toutefois un caractère transitoire compte tenu de la conductivité finie du liquide et du solide. Si par ailleurs, le liquide est soumis à un champ continu, l'échantillon pyroélectrique se trouve soumis à un couple transitoire. Ce couple a été mesuré directement à l'aide d'une balance de torsion sur un échantillon de niobate de lithium plongé dans l'huile. Le transitoire observé est en accord avec un modèle semi-quantitatif impliquant la diffusion thermique et la relaxation diélectrique liée à la conduction ohmique. L'application du phénomène à la fabrication de composite piézoélectrique est brièvement signalée.
\end{abstract}

Abstract. - A pyroelectric sample suddenly dipped into a hot liquid dielectric displays an electric dipole moment which however has a transient character due to the finite conductivities of both liquid and solid media. If in addition, the liquid is subjected to a DC electric field the pyroelectric sample suffers a transient torque. This torque was measured directly with a torsion pendulum using lithium niobate as the pyroelectric and oil as a liquid dielectric. The observed transient was shown to be consistent with a simple semiquantitative model involving heat diffusion and dielectric relaxation via ohmic conduction. Application of this effect to the fabrication of piezoelectric composites is briefly mentionned.

\section{Introduction.}

Piezoelectric composites are usually made of piezoelectric inclusions imbedded in a polymer matrix. The basic problem with these materials then regards the mutual orientation of those piezoelectric grains. For instance if the grains are polar (pyroelectric), their polar axes should be all pointing toward the same direction at least approximately, otherwise no macroscopic piezoelectric property would be obtained. One solution is to use inclusions that are not only pyroelectrics but also ferroelectrics [1]. Once fabricated, such a composite will display piezoelectricity only after poling just like - but not so easy as - the well known PZT ceramics.

Other solutions have been independently proposed by Zipfeld Jr. [2] and Peuzin [3]. Both require only pyroelectric (not necessarily ferroelectric) grains and relie on the same basic principle. The process of reference [3] is illustrated in figure 1. A heated vessel contains an insulating liquid which is actually a pre polymer material. A DC electric field $\mathbf{E}$ is applied to the liquid thanks to a pair of immersed electrodes. A powder feeder regularly

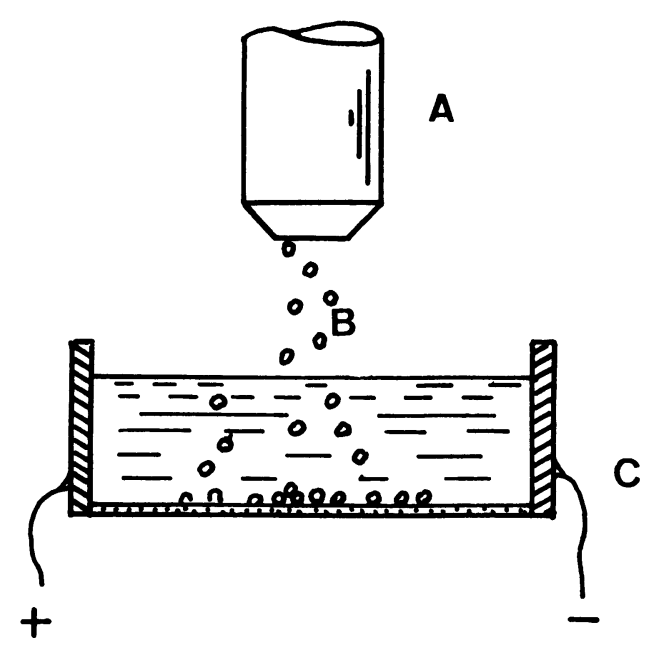

Fig. 1. - Illustration of the pyroelectric orientation process: A powder feeder; $\mathbf{B}$ pyroelectric grains; C prepolymer liquid in applied electric field.

drops pyroelectric grains into the liquid. When contacting the bath each particle undergoes a sudden temperature rise $\Delta T$, and as a consequence of the 
pyroelectric effect, gets a dipole moment $\mathbf{D}$ which tends to align itself with field $\mathbf{E}$. This occurs via mechanical rotation of the grain in the liquid since $\mathbf{D}$ is rigidly bound to the crystal lattice. It is thus expected that a deposit of oriented grains can be obtained at the bottom of the vessel. This situation is then frozen in by solidification of the polymer, producing the requested composite.

This work was aimed at proving the feasibility of the pyroelectric orientation process just described. The paper is composed of two parts : In the first one, a simple theory of the transient pyroelectric torque is given. The second part is devoted to a direct experimental verification of this theory. In our conclusion we also give a very brief account on the actual fabrication of composites using this process.

\section{Pyroelectric transient torque. Theory.}

We consider a pyroelectric grain of ellipsoidal shape whose polar axis $\mathrm{O} x$ is assumed to be along one of the ellipsoid principal directions. Let $\varepsilon_{1}$ and $\rho_{1}$ respectively be the dielectric constant and the resistivity along $\mathrm{O} x$ and $p$ the pyroelectric coefficient. We further assume that the grain is surrounded by an infinite isotropic dielectric characterized by $\varepsilon_{2}$ and $\rho_{2}$.

Though the polar grain has a spontaneous polarization $P$, its net dipole moment, at a stable temperature and in zero applied field, is expected to be zero because of the finite conductivity of both media : The corresponding equilibrium situation is illustrated in figure 2 . The bound charges due to the discontinuity of the normal component of $P$ at the grain boundary are canceled out by free charges which have been carried here through the conduction mechanism.

We now assume that at time $t=0, P$ becomes an arbitrary function of time $t$. The equilibrium is thus broken and the grain is expected to carry an effective dipole moment $D(t)$ which we wish now to calculate.

As shown in appendix $1, D$ obeys the following differential equation

$$
\begin{aligned}
{\left[(1-A) \varepsilon_{2}\right.} & \left.+A \varepsilon_{1}\right] \frac{\partial D}{\partial t}+ \\
& +\left[\frac{(1-A) \varepsilon_{2}}{\tau_{2}}+\frac{A \varepsilon_{1}}{\tau_{1}}\right] D=v \frac{\partial P}{\partial t}
\end{aligned}
$$

where $A$ is the depolarizing field coefficient of the ellipsoid along the material polar axis $\mathrm{O} x$ multiplied by the vacuum permittivity $\varepsilon_{0} . v$ is the ellipsoid volume. For a step like increase $\overline{\Delta P}$ of $P$ at $t=0$, the solution of (1) is

$$
D=\frac{\overline{\Delta P} v}{(1-A) \varepsilon_{2}+A \varepsilon_{1}} \exp (-t / \tau)
$$

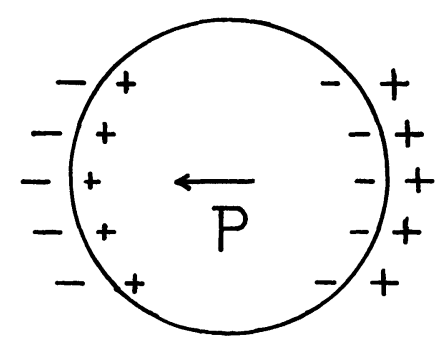

Fig. 2. - Electrostatic equilibrium situation of a spontaneously polarized grain of finite resistivity.

with

$$
\begin{gathered}
\tau=\frac{A \varepsilon_{1}+(1-A) \varepsilon_{2}}{A \varepsilon_{1} / \tau_{1}+(1-A) \varepsilon_{2} / \tau_{2}} ; \\
\tau_{1}=\varepsilon_{0} \varepsilon_{1} \rho_{1} ; \quad \tau_{2}=\varepsilon_{0} \varepsilon_{2} \rho_{2}
\end{gathered}
$$

therefore the dipole moment actually has a finite life time $\tau$ due to the conductivity of both media.

The mechanical torque $\zeta$ produced by a field $\mathbf{E}$ on a dipole with effective moment $\mathbf{D}$, inside a liquid dielectric with dielectric constant $\varepsilon_{2}$, has been calculated by several authors [4]. When $\mathbf{D}$ and $\mathbf{E}$ are perpendicular to each other, the result is

$$
\zeta=\varepsilon_{2} E D .
$$

Since this result relies on the correct definition of effective dipole moment $D$, we found worthwhile, for self consistency reasons, to give another demonstration of this result in appendix 2 .

In case of a step like increase of the polarization (see relation (2)) we get

$$
\zeta=\frac{\varepsilon_{2} v \overline{\Delta P} E}{(1-A) \varepsilon_{2}+A \varepsilon_{1}} \exp (-t / \tau) .
$$

However, in the experiments to be described below the polarization jump $\Delta P$ is not instantaneous. $\Delta P(t)$ is rather the solution of a heat diffusion equation which leads to a finite rise time of the polarization increase. Since we are only interested in semiquantitative results, we simply put

$$
\Delta P=p \Delta T\left[1-\exp \left(-t / \tau_{\mathrm{d}}\right)\right]
$$

where $\tau_{\mathrm{d}}$ is the rise time of the sample mean temperature. $\Delta T$ is the total temperature rise of the sample. We easily solve (1) with (6) as a source term and find

$D=\frac{p v \Delta T}{(1-A) \varepsilon_{2}+A \varepsilon_{1}}\left[\exp \left(-t / \tau_{\mathrm{d}}\right)-\exp (-t / \tau)\right]$

and

$\zeta=\frac{\varepsilon_{2} E p v \Delta T}{(1-A) \varepsilon_{2}+A \varepsilon_{1}}\left[\exp \left(-t / \tau_{\mathrm{d}}\right)-\exp (-t / \tau)\right]$. 
For a convenient comparison with our experiments, we first transform (8) into the following reduced form

$$
\frac{\zeta}{\zeta^{*}}=\frac{\exp \left(-t / \tau_{\mathrm{d}}\right)-\exp (-t / \tau)}{\exp \left(-t^{*} / \tau_{\mathrm{d}}\right)-\exp \left(-t^{*} / \tau\right)}
$$

where $\zeta^{*}$ is the maximum torque assumed to be reached at time $t^{*}$. It is easy to show that

$$
t^{*}=\tau_{\mathrm{d}} \frac{\ln \left(\tau_{\mathrm{d}} / \tau\right)}{\tau_{\mathrm{d}} / \tau-1}
$$

and

$$
\zeta^{*}=\alpha \cdot \varepsilon_{2} \cdot E \cdot p \cdot v \cdot \Delta T \cdot m(\tau)
$$

where

$$
\alpha=\frac{1}{\varepsilon_{2}(1-A)+\varepsilon_{1} A}
$$

and

$$
m(\tau)=\frac{\exp \left(-t^{*} / \tau_{\mathrm{d}}\right)-\exp \left(-t^{*} / \tau\right)}{\tau_{\mathrm{d}} / \tau-1}
$$

\section{Pyroelectric transient torque. Experiment.}

The pyroelectric transient torque was experimentally measured with the arrangement depicted in figure 3.

A pyroelectric " grain », actually a lithium niobate disk (thickness $2 \mathrm{~mm}$, diameter $5 \mathrm{~mm}$ ) was glued on the axis of a vertical torsion pendulum. The disk axis, also the material pyroelectric axis, was perpendicular to the pendulum rotation axis and could thus rotate inside an horizontal plane. In its «standby " position, the disk was maintained in air between two parallel and vertical copper plates. Flowing water was used to keep the plates temperatures $T_{\mathrm{A}}$ at or slightly below room temperature, hence the name " cold cell » for this part of the device. We expected the sample temperature to be close to $T_{\mathrm{A}}$ in its standby position. A « hot cell » was put just below the cold one. It was actually an oil filled vessel with means to apply an horizontal DC field and to heat the oil bath at a prescribed temperature $T_{\mathrm{H}}>T_{\mathrm{A}}$ (see Fig. 3 for details). The direction of the DC field in the horizontal plane was perpendicular to the polar axis of the disk sample in its standby position.

In order to measure the transient torque, both cells - which were rigidly bound together - were suddenly raised until the sample was totally immersed in the oil bath. This was done thanks to a mechanical system which is not depicted in figure 3 .

The resulting torque produced an angular deflection of the torsion pendulum which could be measured with an optical lever system and a position sensitive photodetector. This deflection was kept small (less than $2^{\circ}$ ) so that $\mathbf{D}$ and $\mathbf{E}$ could always be assumed to be perpendicular to each other in
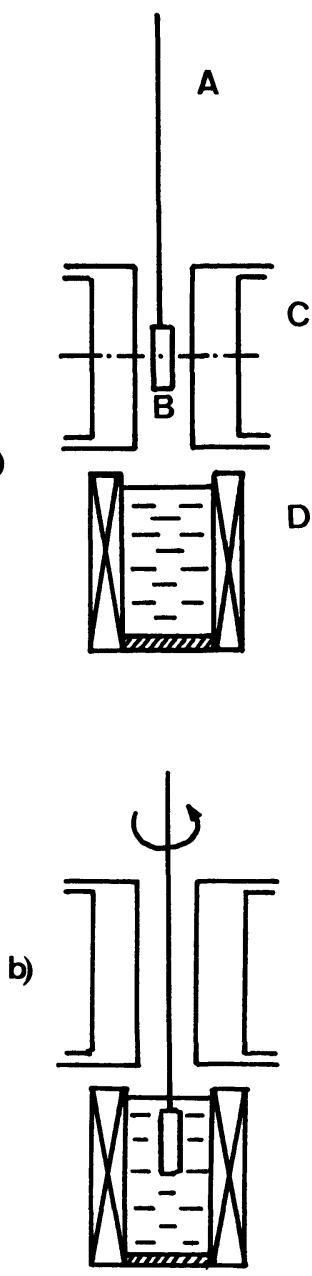

Fig. 3. - Experimental arrangement for studying pyroelectric transient torque. A vertical torsion pendulum, B lithium niobate disk inside cold cell C. D hot cell filled with oil, d.c electric field is applied normally to the picture plan (electrodes not shown). a) sample in standby position, b) sample in measurement position.

compliance with theoretical assumptions. The torque transients were directly recorded on an analog plotter as a function of time $t$.

Figure 4 shows a typical result. As expected from relation (8), the torque starts from zero, goes through a maximum and then falls back to zero. Note the torque scale $\left(\sim 10^{-6} \mathrm{~N} . \mathrm{m}\right)$ and the time scale $(\sim \mathrm{s})$. A lot of such transients were recorded with different temperature steps $\Delta T=T_{\mathrm{H}}-T_{\mathrm{A}}$ and different DC fields $E$.

An independent experiment was needed to determine the parameters, $p$ (although it can in principle be found in the litterature) and particularly the temperature rise time $\tau_{\mathrm{d}}$ : One of the disk sample was fitted with silver paste electrodes and connected to a charge amplifier through a miniature coaxial cable. Thermal conduction through this cable could be assumed negligible. The sample, initially at room temperature, was dipped into an oil bath heated at a 


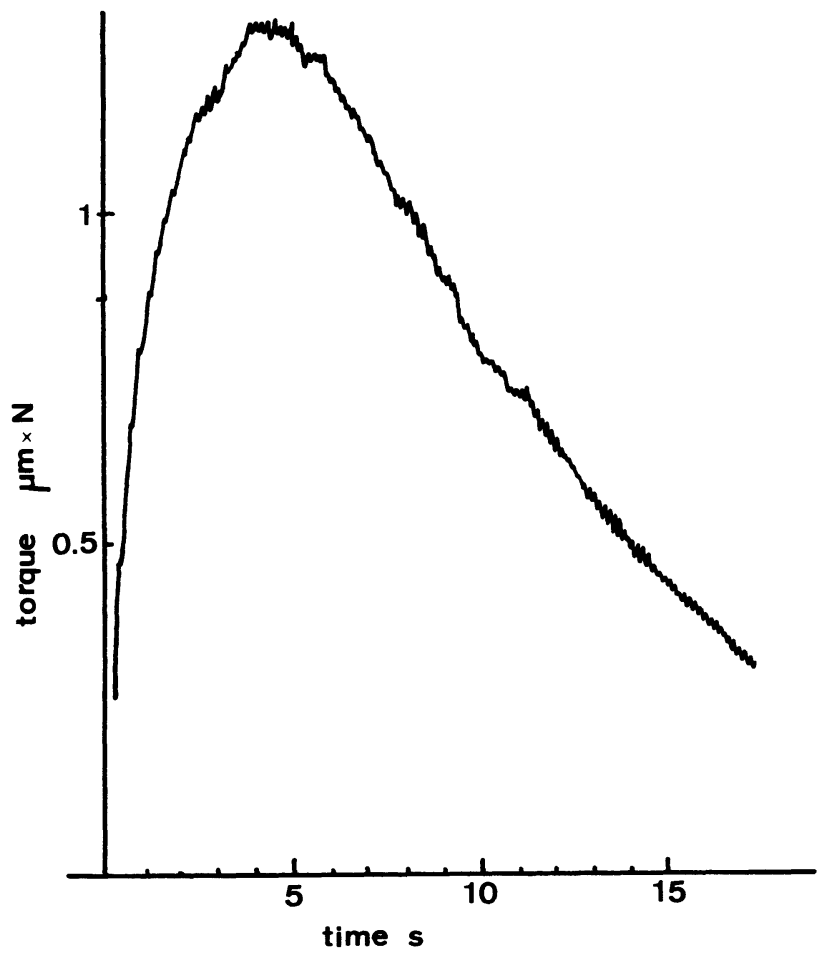

Fig. 4. - A typical torque transient. Superimposed small oscillations are due to insufficient damping of torsion pendulum.

given temperature and the resulting pyroelectric charge was recorded as a function of time. As shown in figure 5 the observed charge transient and hence the sample mean temperature transient is close to the assumed exponential form (see relation (6)). From this measurement one could easily extract

$$
p=8 n C / \mathrm{cm}^{2} \mathrm{~K} ; \quad \tau_{\mathrm{d}}=5,8 \mathrm{~s} .
$$

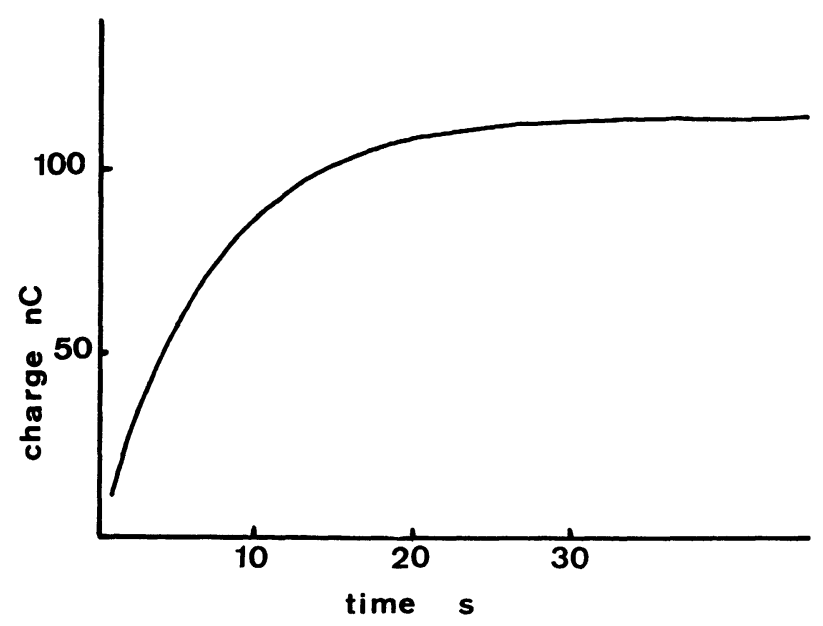

Fig. 5. - Measurement of pyroelectric coefficient $p$, and diffusion time constant $\tau_{\mathrm{d}}$ in oil (sample is a lithium niobate disk, diameter $5 \mathrm{~mm}$, thickness $2 \mathrm{~mm}$, temperature rise is $75 \mathrm{~K}$ ).
Then the comparison of our torque experiments with the simple theory developped in the previous section could be made in two steps.

First the experimental and theoretical transient shapes were compared. This is illustrated by figure 6: The solid curves correspond to theoretical relation (9) with $\tau_{\mathrm{d}}=5,8 \mathrm{~s}$ (as measured above) and with various values of $\tau$. The experimental points were obtained by transforming the experimental transients into their " reduced" form, i.e. by dividing measured value of $\zeta(t)$ belonging to a given transient by the maximum value of $\zeta$ in this transient. Figure 6 deserves some comments. As can be seen there is only qualitative agreement between theory and experiments. Two independant types of disagreement show up. First there is a definite influence of the DC field on the transient shape which naturally is not accounted for by the simple linear theory. Note however that this seems to be true only at low applied field and in the falling part of the transient. This effect is not understood. It is tentatively suggested that it could be due to some kind of non linear conduction in oil or in $\mathrm{LiNbO}_{3}$ or even at the liquid-solid interface. Since this non linearity appears at low field it must be of the "threshold type " rather than of the "saturation type".

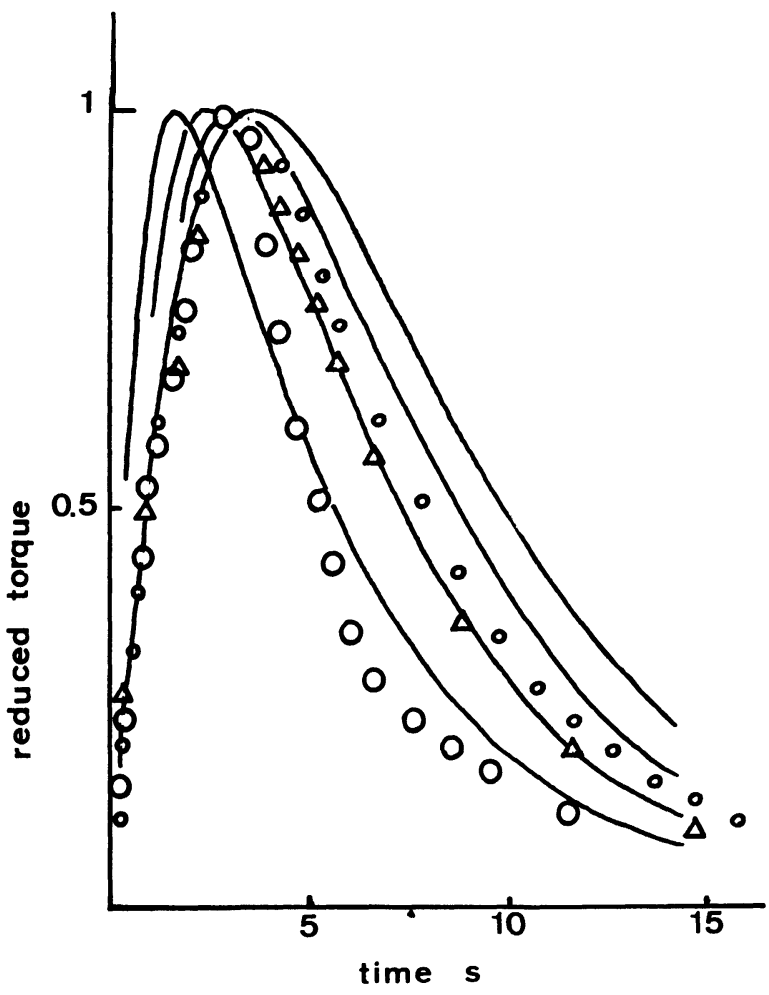

Fig. 6. - Comparison of theoretical and observed transient shapes. Solid curves from left to right : Theoretical transients for respectively $\tau=1,2,3$ and $4 \mathrm{~s}$. Circles and triangles : Experimental points for a temperature rise of $80 \mathrm{~K}$ and various electric fields $E$. Big circles $E=0.5 \mathrm{kV} / \mathrm{cm}$, triangles $E=1.5 \mathrm{kV} / \mathrm{cm}$, small circles $E=2.5 \mathrm{kV} / \mathrm{cm}$. 
If we ignore the low field transient of figure 6 we are left with a second type of disagreement: The rising part and the falling part of the experimental curves do not fit simultaneously with the same theoretical transient. More accurately speaking the value of $\tau$ seems to be higher at the beginning of the transient than at its end. This effect is much easier to interpret. We believe it to be due to the variation of material resistivity $\rho_{1}$ with temperature. At the beginning of the transient, sample temperature is still low and therefore its resistivity still high, giving a large time constant $\tau$ (see relations (3)). At the end of the transient sample temperature is high leading to a decreased resistivity and a smaller value of $\tau$.

In a second step we tried to compare the variation of maximum torque within a given transient, with experimental conditions. This is illustrated in figure 7 which gives maximum torque as a function of the quantity $E . \Delta T . m(\tau)$. The factor $m(\tau)$ has been defined in the theoretical section (see formula (13)). The needed value of $\tau$ is extracted from a comparison of experimental and theoretical transient shape. For instance in the experimental transient of figure 6 we take $\tau=2,5 \mathrm{~s}$ since it gives the best fit with theoretical shape (we ignore low field transient and put emphasis on the falling part of the curve).

This procedure leads to the experimental points drawn in figure 7. Not unexpectedly, the data are highly scattered, but again there is semiquantitative agreement with theory: The dashed lines $a, b, c$ in figure 7 are the theoretical plots corresponding to relation (11) with three different grain geometries. The geometry effect arises through the reduced depolarizing field factor $A$ (see relation (12)). Curve a) stands for a thin plate geometry whereas c) stands

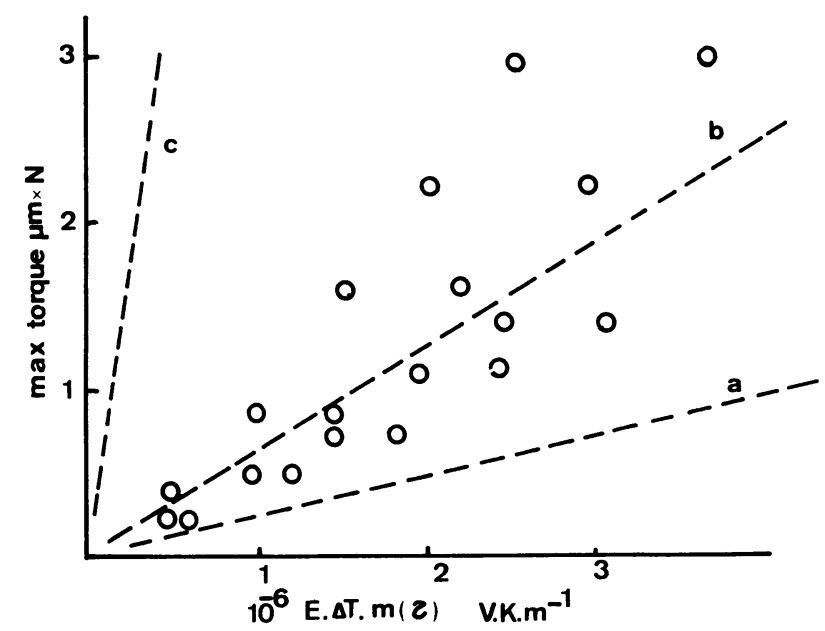

Fig. 7. - Maximum torque as a function of the product $m . E . \Delta T$ where $E$ is the applied field, $\Delta T$ the temperature rise, and $m$ the theoretical normalization factor. Broken lines: theoretical relations for: a) thin plates; b) spheres, c) needles. Circles : experimental points. for a needle like sample. Curve b) stands for the intermediate spherical case. Our experimental results seem to fit approximately with the sphere case, which is not too surprising when looking at our disk aspect ratio $(5 / 2)$.

\section{Conclusion.}

The feasibility of a pyroelectric orientation process was demonstrated: a pyroelectric particle falling into a hot liquid dielectric exhibits a transient dipole moment that depends on particle geometry and dielectric characteristics of liquid and solid. When a DC field is applied to the liquid the particle suffers a pyroelectric transient torque, hence the name of the process.

A simple model was shown to give a good semiquantitative description of experimental results.

Although the fabrication of composite was not described in the paper, it is worthwhile mentionning here that "model composites" were actually fabricated using the pyroelectric process. The grains were lithium niobate needles $\left(3.5 \times 1.2 \times 1 \mathrm{~mm}^{3}\right)$ with the polar axis along needle length. Polystyrene was used as a matrix. Composites were fabricated under the following conditions: temperature of liquid during the orientation process, $100^{\circ} \mathrm{C}$ $\left(\Delta T \simeq 80^{\circ} \mathrm{C}\right)$. Field applied to the liquid ; $6.5 \mathrm{kV} / \mathrm{cm}$. Filling factor, 0.55 . Our measurements of piezoelectric modulus $d_{33}$ on such composites gave values that amounted up to 0.6 times the single crystal value. We believe this to indicate a fairly good efficiency of the process.

\section{Acknowledgements.}

Thanks are due to A. Hugon for her help in the experiments and to C. Castera for her help in the preparation of the manuscript.

\section{Appendix 1.}

\section{Pyroelectric particle in a dielectric matrix.}

Consider a dielectric inclusion of ellipsoidal shape with principal axes $\mathrm{O} x, \mathrm{O} y, \mathrm{O} z$ inside an isotropic infinite dielectric matrix. The particle may be anisotropic, but we assume its dielectric principal axes to be again $\mathrm{O} x \mathrm{O} y \mathrm{O} z$. Let $\varepsilon_{1}$ be the inclusion dielectric constant along $O x$ and $\varepsilon_{2}$ be the matrix dielectric constant. Finally the inclusion is supposed to be pyroelectric with polar axis $O x$. In a pyroelectric experiment the particle actual polarization change $\Delta P_{\text {a }}$ necessarily writes

$$
\Delta P_{\mathrm{a}}=\Delta P+\left(\varepsilon_{1}-1\right) \varepsilon_{0} E_{1}
$$

where $\Delta P$ is the spontaneous polarization change and $E_{1}$ a « reaction field". For symmetry reasons $\Delta P, \Delta P_{a}$ and $E_{1}$ are colinear and along $O x$, hence the 
purely scalar form of the above relation. $\Delta P$ is given by

$$
\Delta P=p \Delta T
$$

where $p$ is the pyroelectric coefficient and $\Delta T$ the temperature change of the inclusion. The reaction field $E_{1}$ has necessarily the following form

$$
E_{1}=-\frac{N}{\varepsilon_{0}} \Delta P_{\mathrm{a}}
$$

It is surpprising that the expression of $N-$ as this coefficient has been defined above - is not easy to find in textbooks. What is rather found is the following expression [5]

$$
E_{1}=\frac{\varepsilon_{2} E}{\varepsilon_{2}+\left(\varepsilon_{1}-\varepsilon_{2}\right) A}
$$

which gives the inclusion internal field $E_{1}$ as a function of an externally applied uniform field $E$, in absence of pyroelectric or any other non electrically induced polarization effect. $A$ is such that $A / \varepsilon_{0}$ would be the depolarizing field coefficient of the inclusion along $\mathrm{O} x$ if it were placed in vacuum. $A$ depends only on the "aspect ratio " of the ellipsoid. (See Osborn [6].)

In order to calculate $N$ we imagine the following experiments :

1) Let $E=0$ and force the inclusion polarization to be $P_{1}$.

Then from the definition of $N$ the resulting internal field $E_{1}(1)$ is

$$
E_{1}(1)=-\frac{N}{\varepsilon_{0}} P_{1}
$$

2) Set $P_{1}=0$ and $E \neq 0$, then we have immediately from (17) by putting $\varepsilon_{1}=1$

$$
E_{1}(2)=\frac{\varepsilon_{2} E}{\varepsilon_{2}+\left(1-\varepsilon_{2}\right) A} .
$$

Now adding those two equilibrium states, we get

$$
E_{1}(3)=E_{1}=\frac{\varepsilon_{2} E}{\varepsilon_{2}+\left(1-\varepsilon_{2}\right) A}-\frac{N}{\varepsilon_{0}} P_{1} \text {. }
$$

This is naturally true for any value of $P_{1}$. We may choose

$$
P_{1}=\varepsilon_{0}\left(\varepsilon_{1}-1\right) E_{1}
$$

in which case we get

$$
E_{1}\left[1+N\left(\varepsilon_{1}-1\right)\right]=\frac{\varepsilon_{2} E}{\varepsilon_{2}+\left(1-\varepsilon_{2}\right) A} .
$$

But since in this case, the inclusion polarization is only field induced, we must also verify relation (17).
We therefore get

$$
\begin{aligned}
1+N\left(\varepsilon_{1}-1\right) & =\frac{\varepsilon_{2}}{\varepsilon_{2}+\left(1-\varepsilon_{2}\right) A} \frac{E}{E_{1}}= \\
& =\frac{\varepsilon_{2}+\left(\varepsilon_{1}-\varepsilon_{2}\right) A}{\varepsilon_{2}+\left(1-\varepsilon_{2}\right) A}
\end{aligned}
$$

which finally gives

$$
N=\frac{A}{\varepsilon_{2}+\left(1-\varepsilon_{2}\right) A}=\frac{A}{A+(1-A) \varepsilon_{2}} .
$$

Define now an effective polarization $\Delta P_{\mathrm{e}}$ such that

$$
E_{1}=-\frac{A}{\varepsilon_{0}} \Delta P_{\mathrm{e}}=-\frac{N}{\varepsilon_{0}} \Delta P_{\mathrm{a}}
$$

The significance of $\Delta P_{\mathrm{e}}$ is the following: It is the particle polarization that would produce the actually observed field if the particle was placed in vacuum. As is shown in appendix 2 , the dipole moment that serves to calculate the torque is $v \Delta P_{\mathrm{e}}$ where $v$ is the particle volume. Now, from (14) and (16) we get

$$
\Delta P_{\mathrm{a}}\left(1+N\left(\varepsilon_{1}-1\right)\right)=\Delta P
$$

and since from (24)

$$
\Delta P_{\mathrm{a}}=\Delta P_{\mathrm{e}} \frac{A}{N}
$$

we get

$$
\Delta P_{\mathrm{e}}\left(\frac{A}{N}+A\left(\varepsilon_{1}-1\right)\right)=\Delta P
$$

Expliciting $N$ we obtain

$$
\Delta P_{\mathrm{e}}\left(A \varepsilon_{1}+(1-A) \varepsilon_{2}\right)=\Delta P .
$$

Multiplying both sides of relation (28) by the particle volume $v$, we get

$$
D\left(A \varepsilon_{1}+(1-A) \varepsilon_{2}\right)=v \Delta P
$$

where $D$ is the effective dipole moment of the particle. So far we have considered only perfectly insulating dielectrics. We now introduce finite resistivities respectively $\rho_{1}$ and $\rho_{2}$ in the particle, and in the matrix.

If we take the Laplace transforms $L D$ and $L \Delta P$ of time functions $D(t)$ and $\Delta P(t)$ respectively, then we can still use relation (29) with $\varepsilon_{1}$ and $\varepsilon_{2}$ replaced by $\varepsilon_{1}+1 / \rho_{1} \varepsilon_{0} u$ and $\varepsilon_{2}+1 / \rho_{2} \varepsilon_{0} u$ respectively. Here $u$ is the I aplaci lariable

Relation (29) then becomes.

$$
\begin{array}{r}
u L D\left(A \varepsilon_{1}+(1-A) \varepsilon_{2}\right)+L D\left(\frac{A}{\varepsilon_{0} \rho_{1}}+\frac{1-A}{\varepsilon_{0} \rho_{0}}\right)= \\
=u v L \Delta P \quad(30)
\end{array}
$$


which is equivalent to the following differential equation :

$$
\begin{array}{r}
\left(A \varepsilon_{1}+(1-A) \varepsilon_{2}\right) \frac{\partial D}{\partial t}+\left(\frac{A \varepsilon_{1}}{\tau_{1}}+\frac{(1-A)}{\tau_{2}} \varepsilon_{2}\right) \\
D=v \frac{\partial \Delta P}{\partial t}=v \frac{\partial P}{\partial t}
\end{array}
$$

with $\tau_{1}=\rho_{1} \varepsilon_{1} \varepsilon_{0} ; \tau_{2}=\rho_{2} \varepsilon_{2} \varepsilon_{0}$.

\section{Appendix 2.}

The field produced by the pyroelectric particle at a distance $r$ much larger than particle size has necessarily the form of a dipole field. We define the effective dipole moment $\mathbf{D}$ as the one that would produce in vacuum the same field as is actually observed in the matrix. Consider a point $M$ in the equatorial plane of the dipole, at a distance $r$. The field at $M$ has only a tangential component given by

$$
E^{\prime}=\frac{D}{4 \pi \varepsilon_{0} r^{3}}
$$

If we put at $M$ a point charge $q$, it will suffer a force

$$
q E^{\prime}=\frac{q D}{4 \pi \varepsilon_{0} r^{3}} \text {. }
$$

There will thus exist a reaction torque on the particle given by

$$
q E^{\prime} r=\frac{q D}{4 \pi \varepsilon_{0} r^{2}}
$$

which may also be written

$$
\frac{\varepsilon_{2}}{4 \pi \varepsilon_{0}} \frac{q D}{\varepsilon_{2} r^{2}}=\varepsilon_{2} E D
$$

where $E$ is the field produced by the point charge on the particle. Note that $\mathbf{E}$ is perpendicular to $\mathbf{D}$. Finally a DC field $\mathbf{E}$, whatever its origin, perpendicular to the effective dipole moment $\mathbf{D}$ produces a torque $\zeta$ of magnitude :

$$
\zeta=\varepsilon_{2} E D
$$

\section{References}

[1] Newnham R. E., Skinner D. P., Klicker K. A., Bhalla A. S., Hardinam B., Gururaja T. R., Ferroelectrics 27 (1980) 49.

[2] Zipfeld Jr. G. G., United State Patent n 440705, oct. 4 (1983).

[3] Peuzin J. C., Brevet Français n 2501916 jul. 9 (1984).

[4] When the polarization is only field induced, and when both dielectrics are perfect insulators, formulas giving forces and torques can be found in textbooks (see for example [5]). The case of dielectrics with finite resistivities (but in absence of any pyroelectric effect), and the associated concept of effective dipole has been discussed by JONES, T. B., J. Electrostat. 6 (1979) 69.

[5] Durand E., Electrostatique et Magnétostatique (Masson et Cie Paris) 1953, p. 430.

[6] Osborne J., Phys. Rev. 67 (1945) 351. 